\section{Die Schmelzwärme und die Atomwärme im schmelzflüssigen Bereich von Eisen, Kobalt und Nickel}

\section{Otmar Vollmer, Rudolf Kohlhaas und Martin Braun * \\ Institut für Theoretische Physik der Universität Köln, Abteilung für Metallphysik}

(Z. Naturforschg. 21 a, 181-182 [1966] ; eingeg. am 22. Dezember 1965)

Die bisher veröffentlichten Daten der Schmelzwärme von Eisen, Kobalt und Nickel unterscheiden sich beachtlich und sind teilweise mit großen Fehlergrenzen behaftet. Die gleiche Feststellung betrifft auch die Atomwärme im flüssigen Zustand der drei Metalle. Eine Neubestimmung dieser Größen erschien somit wünschenswert. Das von Braun und KohlhaAs ${ }^{1}$ entwickelte, unter Schutzgas betriebene, adiabatisch arbeitende Hochtemperaturkalorimeter erlaubt eine kontinuierliche Messung der spezifischen Wärme und gleichzeitig eine Ermittlung der Umwandlungswärmen von Metallen im festen Zustand. Dieses Kalorimeter wurde mit einigen Änderungen versehen, die ausführlich bei Vollmer $^{2}$ beschrieben werden und nunmehr auch eine kontinuierliche Messung an flüssigen Metallen ermöglichen.

Für die Untersuchungen standen Eisen-, Kobalt- und Nickelproben verschiedener Reinheit und Herstellungsart zur Verfügung. Ihre Gewichte liegen zwischen 150 und $180 \mathrm{~g}$. Am gleichen Probenmaterial führte KoHLHaAs $^{3}$ Messungen der magnetischen Suszeptibilität durch.

Eisen 1: $99,87 \mathrm{Fe}$; $<0,1 \mathrm{Ni} ;<0,03 \mathrm{Mo}$.

Eisen 2: 99,97 Fe; $0,009 \mathrm{C} ; 0,0029 \mathrm{O}_{2} ; 0,004 \mathrm{~S}$; $0,003 \mathrm{P}$; $0,001 \mathrm{Si} ; 0,002 \mathrm{Al} \mathrm{u.} \mathrm{N}_{2} ; 0,0005 \mathrm{Mn}$.

Kobalt 1: Die Kobaltstange wurde aus spektrographisch untersuchten, 99,999\% reinen Kobaltschwamm-Qualitäten von der Firma Johnson, Matthey \& Co. erschmolzen.

Kobalt 2: 99,5 Co; 0,36 Ni; 0,07 Si; 0,025 C; 0,01 Mn, P, $\mathrm{S}$ u. V; $<0,01 \mathrm{Cr} ; 0,004 \mathrm{O}_{2}$.

Nickel: $\quad 99,98 \mathrm{Ni} ; 0,01 \mathrm{Fe} ; 0,001 \mathrm{Si}, \mathrm{MgO}$ u. CaO.

In Tab. 1 sind die eigenen Meßergebnisse der Schmelzwärmen mit den jeweiligen Fehlergrenzen, die für sämtliche Proben der drei Metalle gelten, sowie vergleichsweise die Daten anderer Autoren zusammengestellt. Die Aufheizgeschwindigkeit betrug bei allen Messungen außerhalb des Umwandlungsbereiches 2-3 $\mathrm{grad} / \mathrm{min}$. Der Schmelzprozeß dauerte ca. $1-2 \mathrm{~h}$ und erstreckte sich über ein Temperaturintervall von ungefähr $10^{\circ}$. Als Schutzgas wurde Argon der Reinheit 99,9993\% verwendet. Selbst nach mehrmaligem Schmel-

* Jetzt: Institut für Anorganische Chemie der Universität Köln.

1 M. Braun u. R. Kohlhaas, Z. Angew. Phys., demnächst.

2 O. Vollmer, Diplomarbeit, Köln 1966.

3 R. Kohlhaas, Arch. Eisenhüttenwes. 36, 437 [1965].

4 A. Ferrier, C. R. Acad. Sci. Paris 254, 104 [1962].

5 A. Ferrier u. M. Olette, C. R. Acad. Sci. Paris 254, 2322 [1962].

\begin{tabular}{|c|c|c|c|}
\hline \multirow{2}{*}{ Autoren } & \multicolumn{3}{|c|}{$\Delta h_{\mathrm{m}}$ in $\mathrm{J} / \mathrm{g}$-Atom } \\
\hline & $\begin{array}{c}\mathrm{E} \text { is e n } \\
\text { bei }\end{array}$ & $\begin{array}{c}\text { Kob a l t } \\
\text { bei }\end{array}$ & $\underset{\text { bei }}{\mathrm{N} \text { i c k e l }}$ \\
\hline $\begin{array}{l}\text { Eigene } \\
\text { Meßergebn. }\end{array}$ & $\begin{array}{l}T=1809^{\circ} \mathrm{K}: \\
14.400 \pm 200\end{array}$ & $\begin{array}{l}T=1767^{\circ} \mathrm{K}: \\
16200 \pm 250\end{array}$ & $\begin{array}{l}T=1725^{\circ} \mathrm{K}: \\
16900 \pm 250\end{array}$ \\
\hline 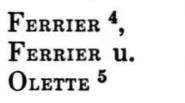 & $\begin{array}{l}T=1809^{\circ} \mathrm{K}: \\
13800 \pm 400\end{array}$ & & \\
\hline $\begin{array}{l}\text { Geoffray, } \\
\text { Ferrier u. } \\
\text { Olette }^{6}\end{array}$ & & & $\begin{array}{l}T=1726^{\circ} \mathrm{K}: \\
17500 \pm 250\end{array}$ \\
\hline $\begin{array}{l}\text { HULtGREN u. } \\
\text { Mitarbeiter }\end{array}$ & $\begin{array}{l}T=1809^{\circ} \mathrm{K}: \\
15200 \pm 2500\end{array}$ & $\begin{array}{c}T=1768^{\circ} \mathrm{K}: \\
17200\end{array}$ & $\begin{array}{c}T=1725^{\circ} \mathrm{K}: \\
17600\end{array}$ \\
\hline KUBASCHEWSKI $^{8}$ & $\begin{array}{l}T=1812^{\circ} \mathrm{K}: \\
15200 \pm 400\end{array}$ & $\begin{array}{l}T=1768^{\circ} \mathrm{K}: \\
15700 \pm 1250\end{array}$ & $\begin{array}{l}T=1728{ }^{\circ} \mathrm{K}: \\
17700 \pm 300\end{array}$ \\
\hline
\end{tabular}

Tab. 1. Die Schmelzwärme $\Delta h_{\mathrm{m}}$ von Eisen, Kobalt und Nickel im Vergleich mit anderen Autoren.

zen ließen sich die Proben ohne große Schwierigkeit aus den Tiegeln entfernen. Sie zeigten keine Reaktionsund Anlaufspuren. Die Gewichtsverluste lagen bei $0,1 \%$. Entscheidend für diese einwandfreie Meßdurchführung dürften die Resistenz der hochreinen $\mathrm{Al}_{2} \mathrm{O}_{3}$-Tiegel gegen die Metallschmelzen und die Verwendung des hochreinen Argons als Schutzgas in Verbindung mit einem geringfügigen im Kalorimeter herrschenden Gasüberdruck gewesen sein.

Des weiteren wurde die Atomwärme im schmelzflüssigen Bereich der drei Metalle ermittelt. Die Ergebnisse sind in Abb. 1 dargestellt (Kurven II, III, V). Sie weisen eine Meßunsicherheit von $\pm 3 \%$ auf. Entscheidend für diese relativ hohe Genauigkeit ist $u$. a. eine möglichst genaue Bestimmung der großen Fremdkapazität, die durch das die Probe umgebende Keramikmaterial verursacht wird. Sie kann im festen Zustand, d. h. vor der Schmelze, experimentell durch Vergleich ermittelt werden, weil hier die Meßergebnisse von Braun und KonlhaAs $^{9,10}$ vorliegen (s. Abb. 1). Die hierbei sich jeweils ergebende Fremdkapazität wird in den schmelzflüssigen Bereich hineinextrapoliert, wobei deren sprunghafte Zunahme beim Schmelzen infolge eines besseren Wärmekontaktes durch Messungen an verschiedenen Proben- und Fremdmassen befriedigend genau feststellbar ist.

In $\mathrm{Abb} .1$ sind weiterhin die von Hultgren, ORR, ANDerson und $\mathrm{K}_{\text {elley }}{ }^{7}$ zusammengestellten Daten der Atomwärme strichpunktiert eingezeichnet (Kurven I, IV, VI). Die Kurven beziehen sich in der Angabe von

6 H. Geoffray, A. Ferrier u. M. Olette, C. R. Acad. Sci. Paris 256, 139 [1963].

7 R. Hultgren, R. L. Orr, Ph. D. Anderson u. K. K. Kelley, Selected Values of Thermodynamic Properties of Metals and Alloys, John Wiley \& Sons, Inc., New York, London 1963.

8 O. Kubaschewski, Z. Elektrochem. 54, 275 [1950].

9 M. Braun u. R. KohlhaAs, Z. Naturforschg. 19 a, 663 [1964].

10 M. Braun u. R. Kohlhaas, Phys. Status Solidi 12,429[1965]. 


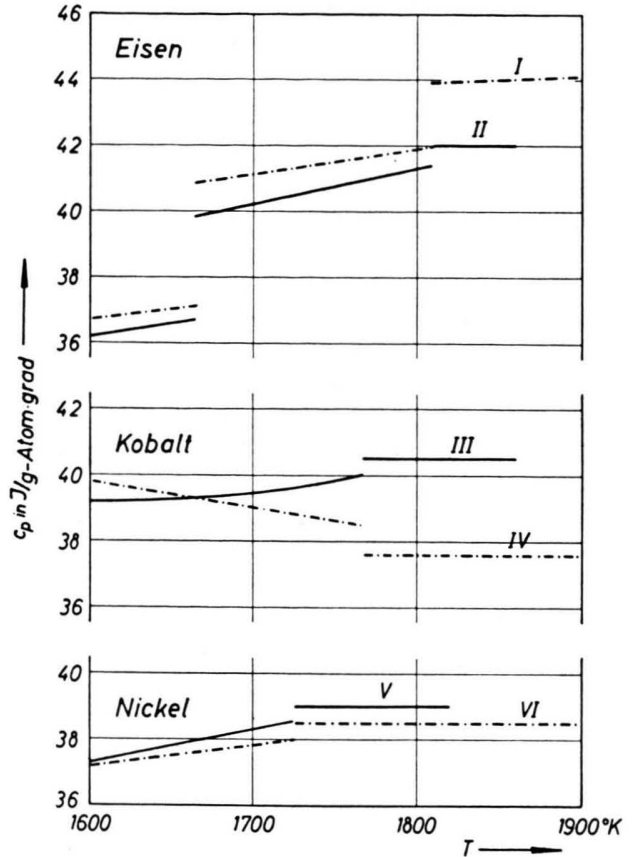

Abb. 1. Die Atomwärme $c_{p}$ von Eisen, Kobalt und Nickel in Abhängigkeit von der Temperatur $T$ ab $1600{ }^{\circ} \mathrm{K}$. Die römischen Ziffern bedeuten: I, IV und VI von Hultgren und Mitarbeitern ${ }^{7}$ ausgewählte Daten; II, III $u$. V eigene Messungen.

$c_{p}$ (liquid) bei Eisen und Nickel auf Wärmeinhaltsmessungen anderer Autoren, bei Kobalt sind es geschätzte Werte.

Zwei Eigenschaften der eigenen Meßergebnisse sind noch besonders erwähnenswert, einerseits die geringe Vergrößerung der Atomwärme beim Übergang fest flüssig, andererseits die Konstanz im flüssigen Bereich, die einer qualitativen Annäherung an das DulongPerirsche Gesetz gleichkommt. Zusätzlich soll bemerkt werden, daß im Vergleich der drei Metalle Eisen, Kobalt und Nickel miteinander die Atomwärme absinkt, während die Schmelzwärme zunimmt.

Tab. 2 gibt die gemessenen Werte der Atomwärme von Eisen, Kobalt und Nickel wieder, ergänzt durch Daten für den festen Zustand der Metalle, die den Arbeiten von Braun und KohlhaAs ${ }^{\mathbf{9}, 10}$ entnommen sind und eine Meßunsicherheit von $\pm 2 \%$ besitzen (in den genannten Veröffentlichungen fehlen zahlenmäßige Angaben für Eisen und Nickel).

11 G. Borelius, Arkiv Fysik 6, 191 [1953].

12 O. Krisement u. F. Wever, Special Issue of Appl. Sci. Res. (Borelius-Festschrift), 249 [1954].

\begin{tabular}{|c|c|c|c|c|}
\hline \multirow{2}{*}{$T$ in ${ }^{\circ} \mathrm{K}$} & \multicolumn{4}{|c|}{$c_{p}$ in $\mathrm{J} / \mathrm{g}$-Atom grad } \\
\hline & & Eisen & Kobalt & Nickel \\
\hline 300 & & 24,8 & 24,9 & 26,3 \\
\hline 400 & & 27,4 & 26,8 & 28,9 \\
\hline 500 & & 29,7 & 28,3 & 31,5 \\
\hline 600 & & 31,9 & 29,6 & 34,9 \\
\hline 631 & & - & - & 39,4 \\
\hline 700 & & 34,3 & 30,7 & 30,8 \\
\hline 800 & & 37,8 & 32,4 & 31,0 \\
\hline 900 & & 42,8 & 34,5 & 31,6 \\
\hline 1000 & & 54,0 & 36,6 & 32,2 \\
\hline 1039 & & 76,0 & - & - \\
\hline 1100 & & 43,9 & 39,1 & 32,9 \\
\hline 1184 & (a) & 39,9 & - & - \\
\hline & $(\gamma)$ & 33,3 & - & - \\
\hline 1200 & & 33,4 & 42,3 & 33,7 \\
\hline 1300 & & 34,1 & 47,0 & 34,5 \\
\hline 1377 & & - & 57,0 & - \\
\hline 1400 & & 34,8 & 43,6 & 35,4 \\
\hline 1500 & & 35,5 & 39,7 & 36,3 \\
\hline 1600 & & 36,3 & 39,2 & 37,3 \\
\hline 1664 & & 36,7 & - & - \\
\hline & $(\delta)$ & 39,8 & - & - \\
\hline 1700 & & 40,2 & 39,5 & 38,3 \\
\hline 1725 & & - & - & 38,5 \\
\hline & & - & - & (l) 39,0 \\
\hline 1767 & & - & (s) 40,0 & - \\
\hline & & - & 40,5 & - \\
\hline 1800 & & 41,3 & 40,5 & 39,0 \\
\hline 1809 & (s) & 41,4 & - & - \\
\hline & (l) & 42,0 & - & - \\
\hline 1820 & & 42,0 & 40,5 & 39,0 \\
\hline 1860 & & 42,0 & 40,5 & - \\
\hline
\end{tabular}

Tab. 2. Die Atomwärme $c_{p}$ von Eisen, Kobalt und Nickel (Mittelwerte).

Mit diesen neuen Meßergebnissen wurde nun versucht, die Frage des strukturellen Zusammenhangs beim Eisen zwischen der Fernordnung der kubisch raumzentrierten $\alpha(\delta)$-Phase und der Nahordnung der flüssigen Phase auf der Grundlage der Boreliusschen Hypothese ${ }^{11}$ zu klären. Im Gegensatz zu Krisement und WEVER ${ }^{12}$ ergaben die eigenen Rechnungen keine Bestätigung der Boreliusschen Theorie und somit keinen Hinweis auf einen strukturellen Zusammenhang zwischen den beiden erwähnten Phasen des Eisens.

Herrn Prof. Dr. Heinrich Lange danken wir recht herzlich für die stete Förderung dieser Arbeit. In dankenswerter Weise stellten uns Herr Dr. F. Assmus, Vakuumschmelze AG-Hanau, Herr Priv.-Doz. Dr. W. A. Fischer, Max-Planck-Institut für Eisenforschung-Düsseldorf, und Herr Dr. H. Preisendanz, Deutsche Edelstahlwerke AG-Krefeld, Probenmaterial zur Verfügung. 